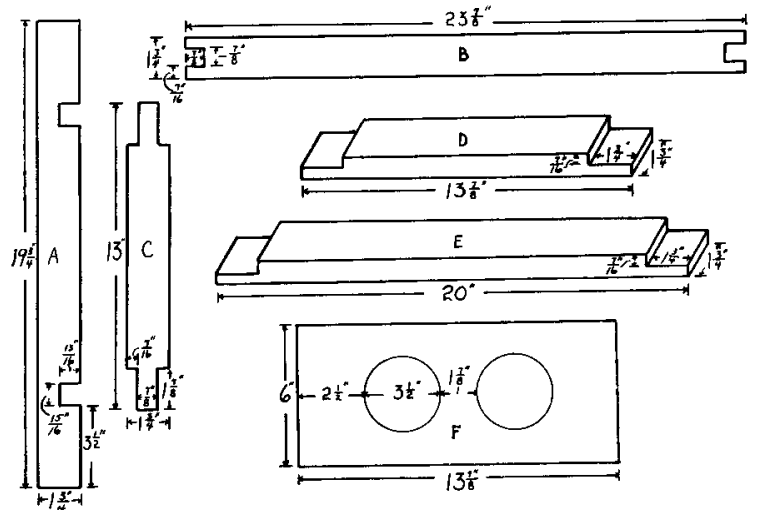

Fic. 2. Wooden parts used in the construction of sea urchin cage. A. End pieces, horizontal (4), B. Long pieces, horizontal (4), C. Vertical end pieces (4), 1). Cross pieces of cover (2), E. Long pieces of cover (2), and F. Inside supports for plastic boxes (2).

the use of these cages, the choice of location is of the utmost importance. Preferably this location should be favorable to the species being studied, not heavily frequented by inquisitive people, and protected from the direct surf. In such a location these cages have been weighted down with rocks, tied together in sets of 3 and then tied to larger rocks. After a year the wood showed some evidence of attack by Limnoria, and the wire was beginning to rust. Even so, it appeared that they could safely have been used for several more months.

In any studies using such cages it is essential to give careful attention to feeding the animals, to removing decomposing food and dying animals, and to keeping the mesh of the cages free of any debris that might impede the free circulation of the water.

It appears likely that cages of this general type should be useful in studies involving other aquatic animals, and it is hoped that their use may result in the accumulation of additional data relevant to the growth rate and longevity of the species studied.

\section{Sum MARY}

The construction of a cage for holding sea urchins and other marine animals during growth experiments has been described. In view of the small amount of available data pertaining to the growth rates of the vast majority of marine invertebrates, the usefulness of data obtainable from caged specimens is discussed.

Department of Zoologiy

EMERY F. SWAN

\section{University of New Hampshire}

Durham, New Hampshire

* In regions of severe shipworm infestation, cages with galvanized iron frames are advisable.

\title{
BEES, DAPHNIA, AND POLARIZED LIGHT
}

\section{INTRODUCTION}

Although a variety of animals, mostly arthropods, have been shown to orient to the polarization plane of polarized light, the means of orientation has been the subject of considerable speculation. Optical and physiological analyses of the visual apparatus by a number of authors have until recently revealed no clear and convincing evidence of a device by which the polarization plane is detected.

This note presents a set of simple experiments that will distinguish which of 3 possible kinds of polarization analyzers is employed by an animal orienting to the polarization plane of overhead light. The 3 kinds of analyzers are characterized by their location and operating principles.

The first of these analyzers is not only extra-ocular but is entirely extra-animal. Polarized light may produce patterns of reflectance and scatter from the substratum and similar patterns of scatter from surrounding water, in which the bright and dark areas can be predicted from Fresnel's equations and from scattering theory (for a lucid discussion of these and other properties of polarized light, including the effect of half-wave plates described below, see Jenkins and White 1958). The intensity variations in these patterns have been shown to be adequate for brightness discrimination and therefore for phototactic responses in arthropods (Baylor and Smith 1953 and in press, Bainbridge and Waterman 1959). Thus photopositive animals restrained from vertical movement may move toward the brighter areas of these patterns, which lie in the axis perpendicular to the polarization plane. Discrimination of the polarization plane in overhead light through the use of such brightness patterns will in this paper be called extraocular polarization analysis.

The 2nd kind of analyzer is intra-ocular and depends on one or more reflections or refractions from lenticular surfaces in the dioptric apparatus as predicted by Fresnel equations. These phenomena may occur in onmatidia with optic axes at a considerable angle to the incident light beams. By various combinations of refraction, reflection, and even scatter in the lenses, a small amount of this off-axis incident light may actually reach the rhabdomeres, where it may be seen as "glare" if the projected visual field of the ommatidia involved is very dark compared with the brightness of the primary light source. The brightness of such glare will vary with the azimuth angle of the ommatidium to the polarization plane, and orientation becomes possible if the animal is able to discriminate such brightness variations. If the glare is primarily refracted to the rhabdomeres its brightness is greatest in ommatidia with optic axes parallel to the polarization plane, least in those perpendicular to the plane. If the glare, following initial refraction, is primarily reflected or scattered internally in the dioptric apparatus to the rhabdomeres, then its brightness is greatest in ommatidia with axes perpendicular to the polarization plane, least in those parallel. This means of polarization discrimination will be called intra-ocular reflection-refraction analysis.

The 3rd way of discriminating the polarization plane requires that the animal detect the plane with those ommatidia looking directly at the light source, much as man can do by rotating a sheet of polaroid. The radial array of rhabdomeres in each ommatidium is presumed to act like a radial or concentric polaroid. The rhab- 
domeres are presumed to be dichroic with parallel fast axes for opposite pairs of the array. This or any equivalent means of discrimination will be called intra-ocular dichroic analysis.

\section{Experiments}

It is possible to infer from the data of a series of behavior experiments which kind of polarization analyzer is employed by the experimental animal. These experiments are conducted in a darkroom on a horizontal arena beneath a vertical rarallel or slightly divergent beam of polarized light. In the basic experiment the arena has a black floor and black, shaded sides so that the animal sees the light overhead, the floor beneath, and very little else. All postulated means by which orientation can occur will operate in this experiment, provided of course that the cooperation of the animal can be secured.

Orientation can be measured by inscribing in the arena 2 sets of parallel lines, one parallel with the polarization plane and one perpendicular to it. In our experiments the lines were one inch apart and formed an 8 -inch square, with at least an inch of unlined arena at the edges. As the atimal moves, one observer counts the lines crossed in one set, while a second observer counts lines crossed in the other set. A measure of the degree of orientation is given by the percentage of one of these counts in the sum of the 2 . The percentage of path vector perpendicular to the polarization plane is given below.

The arena for bees was 10 inches square and $1 / 4$ inch deep, with the lines inscribed on a thin, clean glass cover. The black floor was a dull paper with a slight grain having a reflectance bias. To allow correction for this bias the polaroid was used in 2 positions, parallel and perpendicular to the grain. Light intensity at the arena was about 12 foot candles, and the temperature varied from $22^{\circ}$ to $25^{\circ} \mathrm{C}$. Six bees (Table I) averaged $63 \%$ orientation. The result for each bee is the sum of several trials for each position of the polaroid, and it is obvious from a study of the trial data (Baylor and Smith, in press) that real differences exist between bees; some orient more than others in a given situation.

TABLE I. Bees on dull black paper. Plane of polarization in overhead light is either left-right (A) or front-rear (B) with respect to the observers. Numbers are lines crossed by each bee during several 2 -minute runs and represent inches of path vector left-right (a) and frontrear (b). Percentages are of those portions of the paths that are perpendicular to the polarization plane.

\begin{tabular}{|c|c|c|c|c|c|c|c|}
\hline \multirow[b]{2}{*}{ Bee } & \multicolumn{3}{|c|}{$\begin{array}{c}\text { Polaroid Position } \\
\text { A }\end{array}$} & \multicolumn{3}{|c|}{$\begin{array}{c}\text { Polaroid Po ition } \\
\text { B }\end{array}$} & \multirow{2}{*}{$\begin{array}{c}\text { Average } \\
\%\end{array}$} \\
\hline & $\mathbf{a}$ & b & $\%$ & $\mathbf{a}$ & b & $\%$ & \\
\hline $\begin{array}{l}\text { A. } \\
\text { B. } \\
\text { C. } \\
\text { D. } \\
\text { E. } \\
\text { F. }\end{array}$ & $\begin{array}{r}131 \\
129 \\
170 \\
151 \\
164 \\
99\end{array}$ & $\begin{array}{l}241 \\
255 \\
247 \\
341 \\
302 \\
254\end{array}$ & $\begin{array}{l}64.8 \\
66.4 \\
59.3 \\
69.3 \\
64.8 \\
72.0\end{array}$ & $\begin{array}{l}317 \\
225 \\
216 \\
225 \\
337 \\
246\end{array}$ & $\begin{array}{l}226 \\
159 \\
162 \\
149 \\
203 \\
170\end{array}$ & $\begin{array}{l}58.4 \\
58.6 \\
56.5 \\
60.2 \\
62.4 \\
59.2\end{array}$ & $\begin{array}{l}61.6 \\
62.5 \\
57.9 \\
64.7 \\
63.6 \\
65.6\end{array}$ \\
\hline $\begin{array}{l}\text { Ave....... } \\
\text { Std. Dev... }\end{array}$ & & & $\begin{array}{r}66.1 \\
4.4\end{array}$ & & & $\begin{array}{r}59.2 \\
2.0\end{array}$ & $\begin{array}{r}62.7 \\
2.5\end{array}$ \\
\hline
\end{tabular}

If a dull white arena floor is used, extra-ocular analysis becomes impossible, since dull white surfaces offer a nearly uniform field of brig'atness under polarized light. Intra-ocular reflection-refraction analysis will be impaired by the flood of depolarized reflectance from the floor, although a weak pattern may be discernible in ommatidia looking somewhat above horizontal. Intraocular dichroic analysis, on the other hand, is un isturbed; the overhead light remains as it was over a black floor.

Bees do not orient on white paper (Table II), behavior suggesting the absence of an intra-ocular dichroic analyar in these organisms.

Table II. Bees on white paper or clean mirror. Percentages as in Table $I$. The relative orientation is the ratio of the present orientation, minus $50 \%$, divicled by the black paper orientation of the same bee on Table $\mathrm{I}$, minus $50 \%$. For bees $\mathrm{G}$ through $\mathrm{J}$ the ratio denominator was taken as 12.7 , the average from Table $I$.

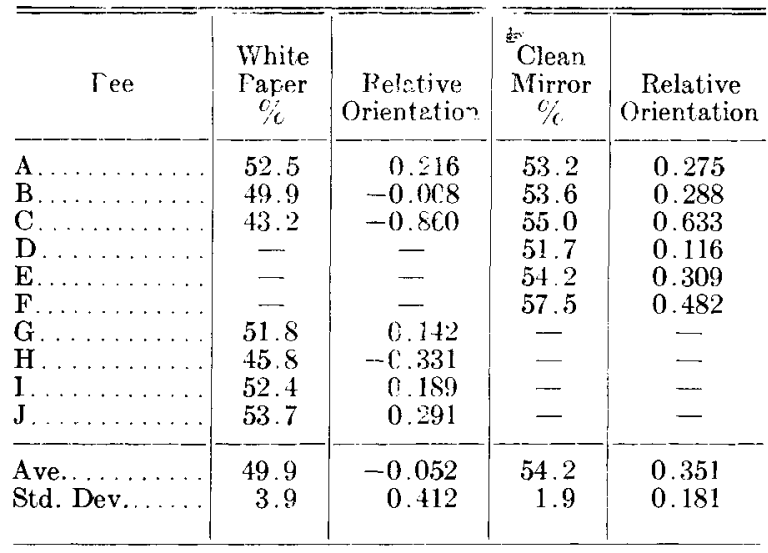

If a clean, front-surface mirror is used instead of a black floor, extra-ocular analysis becomes more difficult. Oblique reflectance to the animal from the peripheral regions of the arena (where the brightness pattern is strongest) is very much less from a mirror than from black paper. Even more important, however, is that its brightness variations are also less. Baylor and Smith (in press) found brightness variations to be only $1 / 3$ as great from a mirror as from black paper, even when expressed in relative terms. On the other hand, the downward and upward directed illumination, fully polarized, vertical, and with a brightness that is independent of the position of the polarization plane, remains ideal for either kind of intra-ocular analysis.

Bees orient only $35 \%$ as well on a mirror as on black paper (Table II). Since this follows closely the weakening of pattern in extra-ocular reflectance, the existence of only an extra-ocular analyzer is suggested.

A more critical test making the same discrimination is based on the effect of half-wave plates. The orientation of an animal on a black surface is first measured, and then a large half-wave plate is inserted just below the polaroid. This rotates the polarization plane $90^{\circ}$ more or less (depending upon wavelength), and the new orientation of the animal is measured. The difference between the 2 orientations is the effect of the large plate. Then the large plate is removed, and a small plate on the end of a thin wire is carefully held over the animal as it moves. This alters the light falling on the animal, but not the light falling on most of the environment. The effect of the small plate on orientation can be compared with the effect of the large plate to determine the relative 
roles of illumination falling directly on the animal and that falling elsewhere on the arena.

A bee selected for very good orientation on black paper (Table III) showed a considerable effect from the large plate, but no significant effect from the small, oneinch square plate. Hence, all of the orientation observed must be ascribed to an extra-ocular analyzer, with the bee responding in a simple way to a brightness pattern in its environment. Although the wave plates were not good (two layers of "Scotch tape"), their imperfection is accommodated in the measures, and the power of this test is very high.

TABLE III. Effect of half-wave plates on a bee selected for good orientation. The relative effect of the small plate is the ratio of the effect of the small plate to the effect of the large wave plate. The standard error of the relative effect was computed 2 ways: as a function of the other errors listed, giving 0.066 , and from 5 estimates of the relative effect using each small plate trial and the nearest trials in time for no plate and for the large plate, giving 0.074 .

\begin{tabular}{|c|c|c|c|c|}
\hline Condition: & $\begin{array}{l}\text { No. } \\
\text { trials }\end{array}$ & Ave. & $\begin{array}{l}\text { Std. } \\
\text { Error }\end{array}$ & Effect \\
\hline $\begin{array}{l}\text { No wave plate (control) } \ldots \\
\text { Small wave plate } . \ldots \ldots \ldots \\
\text { Large wave plate } \ldots \ldots \ldots\end{array}$ & $\begin{array}{r}12 \\
5 \\
8\end{array}$ & $\begin{array}{l}68.1 \\
66.75 \\
47.9\end{array}$ & $\begin{array}{l}1.04 \\
0.87 \\
0.41\end{array}$ & $\begin{array}{l}1.35 \\
20.2\end{array}$ \\
\hline \multicolumn{4}{|c|}{$\begin{array}{l}\text { Relative effect of small wave plate } \ldots \ldots \ldots \ldots \ldots \ldots \\
\text { Standard Error: } \ldots \ldots \ldots \ldots \ldots \ldots \ldots \ldots \ldots\end{array}$} & $\begin{array}{l}0.067 \\
0.070\end{array}$ \\
\hline
\end{tabular}

With aquatic animals the experiments are similar. Daphnia magna were run in a glass dish 12 inches in diameter and 3 inches deep, using $1 \frac{1}{2}$ inches of clarified water. The 2 sets of lines were inscribed on the floor of the dish. An arena floor of beeswax impregnated with carbon black was poured so as to be grainless, and was found to be an excellent dark surface for brightness patterns under polarized light. Black cloth surrounded the dish, whose sides were completely shaded from the light. Daphnids orient very well (Table IV). Although the precentages are much higher than for bees, this is largely a result of the small size of daphnids, which are much less likely than bees to cross lines while turning around.

TABLE IV. Daphnids on black wax or clean mirror surfaces. Percentages and relative orientations as in Tables I and II. Three daphnids used.

\begin{tabular}{|c|c|c|c|}
\hline Daphnid & $\begin{array}{c}\text { Black wax } \\
\%\end{array}$ & $\begin{array}{c}\text { Clean mirror } \\
\%\end{array}$ & $\begin{array}{c}\text { Relative } \\
\text { orientation } \\
\text { using mirror }\end{array}$ \\
\hline $\begin{array}{l}\text { Small. . . . . . . } \\
\text { Medium . . . . } \\
\text { Large ..... }\end{array}$ & $\begin{array}{l}92.2 \\
92.5 \\
86.1\end{array}$ & $\begin{array}{l}94.7 \\
80.7 \\
88.2\end{array}$ & $\begin{array}{l}1.06 \\
0.72 \\
1.06\end{array}$ \\
\hline $\begin{array}{l}\text { Ave.......... } \\
\text { Std. Dev.... }\end{array}$ & $\begin{array}{r}90.3 \\
3.6\end{array}$ & $\begin{array}{r}87.9 \\
7.0\end{array}$ & $\begin{array}{l}0.95 \\
0.20\end{array}$ \\
\hline
\end{tabular}

Using a white paper floor (Table V), 84\% of the orientation persists. This does not necessarily imply the presence of an intra-ocular analyzer, however, since an additional extra-ocular cue is present: scatter in the water. This cue cannot be removed, but it can be made less visible by using a white periphery. Using unshaded white paper around the dish and a black floor, $43 \%$ of the orientation persists. When both the periphery and floor are white, and average of $13 \%$ of the original orientation remains (Table $V$ ) with a considerable variance and no significant differences from zero even at the 0.5 level. It seems reasonable to conclude that the orientation can be eliminated completely if the surroundings of the animal are suitably whitened, even though the overhead polarized light remains in full view, and that an intra-ocular dichroic analyzer is not involved in the orientation of daphnids.

TABLE V. Daphnids on several white backgrounds. The relative orientation compares present orientation, minus $50 \%$, with that on black wax, minus $50 \%$, given in Table IV.

\begin{tabular}{|c|c|c|c|c|c|c|}
\hline Daphnid & $\begin{array}{c}\text { White } \\
\text { floor } \\
\%\end{array}$ & $\begin{array}{c}\text { Rel. } \\
\text { Orient. }\end{array}$ & $\begin{array}{c}\text { White } \\
\text { sides } \\
\%\end{array}$ & $\begin{array}{c}\text { Rel. } \\
\text { Orient. }\end{array}$ & $\begin{array}{c}\text { Both } \\
\text { white } \\
\%\end{array}$ & $\begin{array}{l}\text { Rel. } \\
\text { Orient. }\end{array}$ \\
\hline $\begin{array}{l}\text { Small .... } \\
\text { Medium. } \\
\text { Large. . . }\end{array}$ & $\begin{array}{l}87.0 \\
85.5 \\
78.5\end{array}$ & $\begin{array}{l}0.88 \\
0.84 \\
0.79\end{array}$ & $\begin{array}{l}53.0 \\
87.0 \\
63.0\end{array}$ & $\begin{array}{l}0.07 \\
0.87 \\
0.36\end{array}$ & $\begin{array}{l}69.0 \\
39.5 \\
56.5\end{array}$ & $\begin{array}{r}0.45 \\
-0.25 \\
0.18\end{array}$ \\
\hline $\begin{array}{l}\text { Ave...... } \\
\text { Std. Dev.. }\end{array}$ & $\begin{array}{r}83.7 \\
4.5\end{array}$ & $\begin{array}{l}0.84 \\
0.045\end{array}$ & $\begin{array}{l}68.0 \\
17.0\end{array}$ & $\begin{array}{l}0.43 \\
0.41\end{array}$ & $\begin{array}{l}55.0 \\
15.0\end{array}$ & $\begin{array}{l}0.13 \\
0.35\end{array}$ \\
\hline
\end{tabular}

Using a clean mirror floor (Table IV) orientation is as good as on black paper. This experiment, however, is not as critical for an aquatic animal as for a terrestrial one. The brightness of scatter in the water is about doubled and this may compensate for the decrease in signal from the floor.

The wave plate experiment remains critical. For daphnids, good mica half-wave plates were obtained, and the large plate inserted beneath the polaroid caused a nearly complete reversal of orientation (Table VI).

TABLE VI. Effect of half-wave plates on daphnids, using a black wax surface. Relative effect compares the effect of the small wave plate to that of the large wave plate, using data of Table IV for orientation with no wave plate.

\begin{tabular}{c|c|c|c}
\hline \hline Daphnid & $\begin{array}{c}\text { Small wave } \\
\text { plate \% }\end{array}$ & $\begin{array}{c}\text { Large wave } \\
\text { plate \% }\end{array}$ & $\begin{array}{c}\text { Relative effect } \\
\text { of small plate }\end{array}$ \\
\hline Small...... & 63.5 & 37.6 & 0.53 \\
Medium... & 62.6 & 7.4 & 0.35 \\
Large..... & 57.3 & 43.0 & 0.67 \\
\hline Ave........ & 61.1 & 29.3 & 0.517 \\
Std. Dev.. & 3.3 & 19.2 & 0.161 \\
\hline
\end{tabular}

The small plate (which was a piece of the large one) also had a considerable effect, amounting to $52 \%$ of the effect of the large plate.

These experiments were repeated using a mirror floor. If both intra- and extra-ocular analyzers are involved to account for the partial effect of the small plate, the relative effect of the small plate using a mirror floor should be higher than using a black floor, since the intra-ocular signal is doubled, while, although the scatter signal is doubled, the floor reflectance signal is much reduced. Results were similar, except that the relative effect of the small wave plate was $68 \%$ (Table VI). Using a paired analysis and a one-tailed test, the difference from $52 \%$ is just significant at the 0.05 level. 


\section{Discussion}

Before drawing conclusions several possible artifacts must be examined. Possibly only an intra-ocular analyzer is involved, and the partial effect of the small plate is a result of imperfect alignment while it is held by hand over the animal. To test for the effect of such angular errors, the large plate was inserted 10 degrees out of alignment (with its fast axis $35^{\circ}$, rather than $45^{\circ}$, from the polarization plane). The relative effect, compared with the same plate correctly aligned, averaged $74 \%$ (Table VII). Since this amount of misalignment probably exceeds the maximum momentary deviation of the small plate, and is certainly much greater than the average error, the failure of the small wave plate to produce as great an effect as the large plate cannot be ascribed entirely to position errors.

TABLE VII. Effect of half-wave plates on daphnids, using a clean mirror surface. The first 3 columns are comparable to those of Table VI. The last 2 give only the relative effect of 2 possible artifacts, compared with the effect of the correctly aligned large plate.

\begin{tabular}{l|c|c|c|c|c}
\hline \hline Daphnid & $\begin{array}{c}\text { Small } \\
\text { plate } \\
\%\end{array}$ & $\begin{array}{c}\text { Large } \\
\text { plate } \\
\%\end{array}$ & $\begin{array}{c}\text { Rel. } \\
\text { Effect }\end{array}$ & $\begin{array}{c}\text { Rel. } \\
\text { effect of } \\
\text { Misaligned } \\
\text { large }\end{array}$ & $\begin{array}{c}\text { Rel. } \\
\text { effect of } \\
\text { In active } \\
\text { small }\end{array}$ \\
\hline Small..... & 50.0 & 25.8 & 0.650 & 0.737 & -0.06 \\
Medium. & 40.0 & 15.6 & 0.627 & 0.657 & 0.00 \\
Large..... & 29.7 & 13.1 & 0.777 & 0.830 & 0.13 \\
\hline Ave...... & 39.9 & 18.2 & 0.685 & 0.741 & 0.02 \\
Std. Dev. & 10.2 & 6.7 & 0.081 & 0.087 & 0.06 \\
\hline
\end{tabular}

A second possible artifact lies in the other direction: that in fact the small wave plate has no effect except as a disturbing moving object overhead. This is tested easily by holding the small wave plate over the daphnid in an inactive position (with its fast axis parallel or perpendicular to the polarization plane) so that the light is unaltered although the identical moving object is present. The results (Table VII) show no effect of disturbance.

Another possible explanation for the partial effect of the small wave plate is that the scatter pattern beneath the small plate in the water immediately around the animal is reversed and therefore tends to weaken the scatter pattern in the dish as a whole. The small plate used with daphnids was $3 / 4$ inches square, and the region of illuminated water was 10 inches square. Since the square of distance and area of source for the scatter compensate each other, the brightness pattern is reversed for a daphnid beneath the small plate in $3 / 40$ of the total scatter signal, thus weakening the pattern by $6 / 40$. This cannot possibly account for the average orientation over the mirror under the small wave plate, which was actually reversed so as to be $60.1 \%$ in the axis parallel to th z polarization plane set by the polaroid. To account for this orientation the brightness pattern would have to be reversed in $25 / 40$ of the total signal.

Thus, it can be concluded that daphnids orient through the combined action of an intra-ocular reflection-refraction analyzer and an extra-ocular analyzer, as earlier proposed by Baylor and Smith (1953, and in press), and recently supported by Waterman (1959). Details of the possible intra-ocular mechanism are given by Baylor and Smith (1957).

It can also be concluded that, at least in the experimental conditions used here, bees orient entirely by means of an extra-ocular analyzer. Whether this will be the case for bees in all situations remains to be varified. The naturally darkened surface of a brood comb has been shown to offer brightness patterns of reflectance with variations in intensity that are probably adequate for bees to use (Baylor and Smith, in press). In Stockhammer's (1956) bee-training experiments, the glass covered deep black floors, viewed necessarily at angles close to Brewster's angle by incoming bees, are ideal extra-ocular analyzers. In experiments where the sky is used, it should be remembered that the sky itself has a brightness pattern that probably can be discerned by bees. Such a pattern is a necessary concomitant to a polarization pattern. Wherever in nature a field of polarization is offered to an animal, a pattern of brightness will also be present. Occam's razor dictates that the latter must be eliminated before the former is invoked as an orientation cue.

\section{REFERENCES}

Bainbridge, R. and T. H. Waterman. 1959. Turbidity and the polarized light orientation of the crustacean, Mysidium. J. Exp. Biol. 35: 487-493.

Baylor, E. R. and F. E. Smith. 1953. The orientation of Cladocera to polarized light. Amer. Nat. 87: 98101.

- 1957. Diurnal migration of plankton crustaceans. In: Recent Advances in Invertebrate Physiology. Univ. of Oregon Press.

- In press. Bees and polarized light. In: Symposium on Pattern Recognition (1957). Ed. W. P. Tanner, Jr. Project Michigan Publication.

Jenkins, F. A. and H. E. White. 1958. Fundamentals of Optics. 3rd ed. McGraw-Hill.

Stockhammer, K. 1956. Zur wahrnehmung der schwingungsrightung linear polarisierten lightes bei insecten. Zeit. vergl. Phys. 38: 30-83.

Waterman, T. H. 1959. The problem of polarized light sensivity. XVth International Congress of Zoology, section 6 , paper 37 .

FrEDERICK E. SMith AND

DEPARTMENT OF ZOOLOGY

UNIVERSITY OF MICHIGAN

AND

WoOds Hole Oceanographic Institution

\section{A HOST-PARASITE INTERACTION CONDITIONED BY PREDATION}

The steady densities of interacting populations of Ephestia kühniella (Zell.) and Idechthis canescens (Grav.) in a controlled environment can be markedly changed by manipulating the abundance of the egg-feeding mite Melichares (Blattisocius) tineivorus (Oud.).
In an ecosystem previously described (Flanders 1958), this conditioning of the Ephestia-Idechthis interaction by $M$. tineivorus was strikingly demonstrated. In this system the host and parasite populations are continuously self-perpetuating, apparently infinitely so, having been 